\title{
ON ENTIRE FUNCTIONS OF FAST GROWTH
}

\author{
BYY
}

\author{
S. K. BAJPAI, G. P. KAPOOR AND O. P. JUNEJA
}

ABSTRACT. Let

$$
f(z)=\sum_{n=0}^{\infty} a_{n} z^{\lambda_{n}}
$$

be a transcendental entire function. Set

$$
M(r)=\max _{|z|=r}|f(z)|, \quad m(r)=\max _{n \geqslant 0}\left\{\left|a_{n}\right| r^{\lambda}\right\}
$$

and

$$
N(r)=\max _{n>0}\left\{\lambda_{n}|m(r)=| a_{n}|r| \lambda_{n}\right\} .
$$

Sato introduced the notion of growth constants, referred in the present paper as $S_{q}$-order $\lambda$ and $S_{q}$-type $T$, which are generalizations of concepts of classical order and type by defining

$$
\left.\lambda=\lim _{r \rightarrow \infty} \sup _{(\log }[q] M(r) \mid \log r\right)
$$

and if $0<\lambda<\infty$, then

$$
T=\limsup _{r \rightarrow \infty}\left(\log [q-1] M(r) \mid r^{\lambda}\right)
$$

for $q=2,3,4, \cdots$ where $\log ^{[0]} x=x$ and $\log ^{[q]} x=\log (\log [q-1] x)$. Sato has also obtained the coefficient equivalents of $(* *)$ and $(* * *)$ for the entire function (*) when $\lambda_{n}=n$. It is noted that Sato's coefficient equivalents of $\lambda$ and $T$ also hold true for (*) if $n$ 's are replaced by $\lambda_{n}$ 's in his coefficient equivalents. Analogous to (**) and (***) lower $S_{q^{-o r d e r}} \nu$ and lower $S_{q}$-type $t$ for entire function $f(z)$ are introduced here by defining

$$
\nu=\liminf _{r \rightarrow \infty}(\log [q] M(r) \mid \log r)
$$

and if $0<\lambda<\infty$ then

$$
t=\liminf _{r \rightarrow \infty}\left(\log [q-1] M(r) \mid r^{\lambda}\right), \quad q=2,3,4, \cdots .
$$

For the case $q=2$, these notions are due to Whittakar and Shah respectively. For the constant $\nu$, two complete coefficient characterizations have been found

Received by the editors May 1, 1970 and, in revised form, April 19, 1973. AMS (MOS) subject classifications (1970). Primary 30A66, 30A68.

Key words and phrases. Entire functions, growth estimates, order, lower order, type, lower type, decomposition theorems, Sato $q$ th iterate order, Sato $q$ th iterate lower order, growth numbers, lower growth numbers, coefficient characterizations. 
which generalize the earlier known results. For $t$ coefficient characterization only for those entire functions for which the consecutive principal indices are asymptotic is obtained. Determination of a complete coefficient characterization of $t$ remains an open problem. Further $S_{q}$-growth and lower $S_{q}$-growth numbers for entire function $f(z)$ we defined

$$
\underset{\mu}{\delta}=\lim _{r \rightarrow \infty} \sup _{\text {inf }}\left(\log [q-1] N(r) \mid r^{\lambda}\right),
$$

for $q=2,3,4, \cdots$ and $0<\lambda<\infty$. Earlier results of Juneja giving the coefficients characterization of $\delta$ and $\mu$ are extended and generalized. A new decomposition theorem for entire functions of $S_{q}$-regular growth but not of perfectly $S_{q}$-regular growth has been found.

1. Let $f(z)=\Sigma_{n=0}^{\infty} a_{n} z^{\lambda_{n}}$ be a transcendental entire function. Set the following:

$$
M(r) \equiv M(r, f)=\max _{|z|=r}|f(z)|, \quad m(r) \equiv m(r, f)=\max _{n \geq 0}\left\{\left|a_{n}\right| r^{\lambda}\right\},
$$

and

$$
N(r) \equiv N(r, f)=\max _{n>0}\left\{\lambda_{n}|m(r)=| a_{n} \mid r^{\lambda}\right\} .
$$

$M(r), m(r)$ and $N(r)$ are known as the maximum modulus, maximum term and the rank of the maximum term, respectively, for $f(z)$. It is well known that the functions $\log M(r)$ and $\log m(r)$ are increasing convex functions of $\log r$. Further, it is known that $N(r)$ is an unbounded, nondecreasing step function of $r$ with a left-hand discontinuity at jump points. The values attained by $N(r)$ at these points are called principal indices. We denote by $\left\{\rho\left(n_{s}\right)\right\}$ the jump points of $N(r)$ and its range by $\left\{\lambda_{n_{s}}\right\}$. Following Sato [9], we define

$$
\lambda=\limsup _{r \rightarrow \infty} \frac{\log [q] M(r)}{\log r}
$$

and

$$
T=\limsup _{r \rightarrow \infty} \frac{\log [q-1]}{r^{\lambda}}, \quad q=2,3,4, \cdots,
$$

where $\log ^{[q]} x$ stands for $\log \log \cdots(q$ times $) x$ and (1.2) has a meaning only when $0<\lambda<\infty$. Sato [9] obtained the following coefficient characterization for $\lambda$ and $T$.

THEOREM S. Let $f(z)=\sum_{n=0}^{\infty} a_{n} z^{n}$ be an entire function with the constants $\lambda$ and $T$ as defined in (1.1) and (1.2), then

$$
\lambda=\limsup _{n \rightarrow \infty} \frac{n \log [q-1] n}{\log \left|a_{n}\right|^{-1}}
$$


and

$$
T=\limsup _{n \rightarrow \infty}\left|a_{n}\right|^{\lambda / n} \log [q-2] \frac{n}{e \lambda}
$$

for $q=2,3,4, \cdots$.

Note that, if instead of $f(z)=\sum_{n=0}^{\infty} a_{n} z^{n}$, we choose the entire function $f(z)=\sum_{n=0}^{\infty} a_{n} z^{\lambda_{n}}$, then Theorem $S$ holds true if we replace $n$ by $\lambda_{n}$ in (1.3) and (1.4). The constants $\lambda$ and $T$ are, in fact, the generalizations of the notions of order and type introduced in Valiron [13, p. 34]. We call these constants Sato $q$ th-iterate order and Sato $q$ th-iterate type and, for simplicity, $S_{q}$-order $\lambda$ and $S_{q}$-type $T$. Similar to $S_{q}$-order $\lambda$ and $S_{q}$-type $T$ we introduce the following:

$$
v=\liminf _{r \rightarrow \infty} \frac{\log [q] M(r)}{\log r}
$$

and

$$
t=\liminf _{r \rightarrow \infty} \frac{\log ^{[q-1]} M(r)}{r^{\lambda}} .
$$

We call these constants lower $S_{q}$-order $\nu$ and lower $S_{q}$-type $t$. In fact, these constants are generalizations of the concepts of lower order and lower type introduced respectively by Whittakar [14] and Shah [11]. Whenever $\lambda=\nu, f(z)$ is said to be of $S_{q}$-regular growth and if, in addition $T=t$, it is said to be of perfectly $S_{q}$-regular growth. In case $\nu<\lambda, f(z)$ is of $S_{q}$-irregular growth. We, further, introduce the $S_{q}$-growth number $\delta$ and lower $S_{q}$-growth number $\mu$ for $f(z)$ by the following:

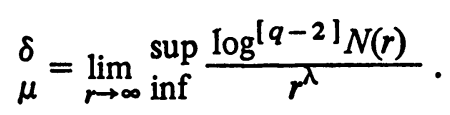

The purpose of the present paper is to investigate the coefficient characterizations of the constants defined above. It will be seen that the results which we obtain generalize and improve considerably the results contained in [1], [5], [10], [11], etc. To avoid unnecessary repetition, we shall denote throughout the paper,

$$
\psi(n)=\left(\lambda_{n+1}-\lambda_{n}\right)^{-1} \log \left|\frac{a_{n}}{a_{n+1}}\right|, \quad \psi\left(n_{k}\right)=\left(\lambda_{n_{k+1}}-\lambda_{n_{k}}\right)^{-1} \log \left|\frac{a_{n_{k}}}{a_{n_{k+1}}}\right|
$$

for the entire function $f(z)=\Sigma_{n=0}^{\infty} a_{n} z^{\lambda_{n}}$.

2. We shall need the following lemmas to prove our theorems in the following sections. Lemmas 1 and 3 generalize the results contained in Valiron [13, p. 33], and Whittakar [14]. Lemmas 4-7 generalize the results contained in [7], $[10],[11]$. 
LEMMA 1. Let $f(z)=\Sigma_{n=0}^{\infty} a_{n} z^{\lambda_{n}}$ be an entire function of $S_{q}$-order $\lambda$ and $S_{q}$-type $T$; then

$$
\limsup _{r \rightarrow \infty} \frac{\log ^{[q]} m(r)}{\log r}=\lambda=\limsup _{r \rightarrow \infty} \frac{\log ^{[q-1]} N(r)}{\log r}
$$

and if $0<\lambda<\infty$, then

$$
T=\limsup _{r \rightarrow \infty} \frac{\log [q-1] m(r)}{r^{\lambda}} .
$$

Proof. (2.1) and (2.2) follow in a straightforward manner by using the following well-known results [13, pp. 31-32].

$$
m(r)<M(r)<m(r)[2 N(r+r / N(r))+1]
$$

and

$$
\log m(r)=\log m\left(r_{0}\right)+\int_{r_{0}}^{r} \frac{N(x)}{x} d x .
$$

LEMMA 2. Let (i) $\log ^{(q-1)} \phi(x)$ be a positive increasing function of $x$;

$$
\liminf _{x \rightarrow \infty} \frac{\log ^{[a-1]} \phi(x)}{\log x}=\alpha \quad(0 \leqslant \alpha<\infty) .
$$

Then, for each pair of positive numbers $\beta, \gamma$ satisfying the inequalities $\alpha<\beta$; $\alpha / \beta<\gamma<1$, there is a sequence $x_{1}, x_{2}, \cdots, x_{n} \rightarrow \infty$ such that

$$
\log ^{[q-2]} \phi(x)<x^{\beta} \quad\left(x_{n}^{\gamma} \leqslant x \leqslant x_{n}\right) .
$$

This lemma is due to Whittakar [14] and so we omit the proof.

LEMmA 3. Let $f(z)$ be an entire function of lower $S_{q}$-order $\nu$ and lower $S_{q}$-type $t ;$ then

$$
\nu=\liminf _{r \rightarrow \infty} \frac{\log ^{[q-1]} N(r)}{\log r}=\liminf _{r \rightarrow \infty} \frac{\log [q]}{\log r}
$$

and, whenever $0<\lambda<\infty$,

$$
t=\liminf _{r \rightarrow \infty} \frac{\log [q-1] m(r)}{r^{\lambda}}
$$

where $\lambda$ denotes $S_{q}$-order of $f(z)$.

Proof. Let

$$
\alpha=\liminf _{r \rightarrow \infty} \frac{\log ^{[q-1]} N(r)}{\log r}
$$


Then, from (2.3) and (2.4) we have $\alpha \leqslant \nu$. Now, let $\alpha<\infty$. By Lemma 2, if $\alpha<\beta, \alpha / \beta<\gamma<1$, then there exists a sequence $R_{1}, R_{2}, \cdots, R_{n} \rightarrow \infty$ for which

$$
\log ^{[q-1]} N(r)<\beta \log r \text { for } R_{n}^{\gamma} \leqslant r \leqslant R_{n} .
$$

Take positive numbers $\delta, \epsilon$ such that $\gamma<\delta<1, \gamma / \delta<\epsilon<1$ and write $S_{n}=R_{n}^{\delta}$ so that $R_{n}^{\gamma}<S_{n}^{\epsilon}<S_{n}<1 / 2 R_{n} \quad\left(n \geqslant n_{1}\right)$. From (2.4) we have for these values of $S_{n}$,

$$
\log m\left(S_{n}\right)<\epsilon N\left(S_{n}\right) \log S_{n}
$$

and

(2.10) $\log m\left(S_{n}\right) \geqslant \log m\left(S_{n}^{\epsilon}\right)+(1-\epsilon) N\left(S_{n}^{\epsilon}\right) \log S_{n}>\frac{1}{\epsilon} \log m\left(S_{n}^{\epsilon}\right)$.

Now by using (2.8), we have for these values of $S_{n}$,

$$
(1-\epsilon) \log m\left(S_{n}\right)<\int_{S_{n}^{\epsilon}}^{S_{n}} \frac{N(x)}{x} d x<(1-\epsilon) \log S_{n} \exp ^{[q-2]} S_{n}^{\beta} .
$$

Thus, from (2.8)-(2.11) we get

$$
\log M\left(S_{n}\right)<\log m\left(S_{n}\right)+\exp ^{[q-3]}\left(2 S_{n}\right)^{\beta}+o(1) .
$$

This implies that

$$
\log M\left(S_{n}\right) \sim \log m\left(S_{n}\right) \quad \text { if } \nu>0 .
$$

In the case when $\nu=0,(2.6)$ and (2.7) are obvious. Further, from (2.10) we have

$$
\log ^{[q]} m\left(S_{n}\right)<(1+o(1)) \log ^{[q-1]} N\left(S_{n}\right) .
$$

From (2.12) and (2.13) the result of the lemma follows.

LEMMA 4. Let $f(z)=\sum_{n=0}^{\infty} a_{n} z^{\lambda_{n}}$ be an entire function of lower $S_{q^{-}}$ order $\nu$; then for an arbitrary sequence of integers $\left\{\lambda_{n_{m}}\right\}$ from $\left\{\lambda_{n}\right\}$ and the corresponding sequence $\left\{a_{n_{m}}\right\}$ from $\left\{a_{n}\right\}$ we have

$$
\nu \geqslant \beta \equiv \liminf _{m \rightarrow \infty} \lambda_{n_{m}} \log ^{[q-1]} \lambda_{n_{m-1}} / \log \left|a_{n_{m}}\right|^{-1} .
$$

Proof. If $\beta=0$, then (2.14) is obvious. Hence, let $0<\beta<\infty$. Then, for given $\epsilon>0, \beta>\epsilon$ and for all $m \geqslant m_{0}(\epsilon)$, we get

$$
\left|a_{n_{m}}\right|>\left[\log [q-2] \lambda_{n_{m-1}}\right]^{-\lambda_{n_{m}} /(\beta-\epsilon)}
$$

\section{Define}




$$
\log r_{n_{m}}=\frac{1}{\beta-\epsilon} \log [q-1] \lambda_{n_{m-1}}+p, \quad p>1, m=1,2, \cdots
$$

Then, for $r_{n_{m-1}} \leqslant r \leqslant r_{n_{m}}$, from (2.15) and (2.16) we get

$$
\begin{aligned}
\log ^{[q]} M(r) & \geqslant \log [q-1]\left\{\lambda_{n_{m}} \log r+\log \left|a_{n_{m}}\right|\right\} \\
& \geqslant \log [q-1]\left\{p \exp ^{[q-2]}\left(r_{n_{m+1}}^{\beta-\epsilon} e^{-p(\beta-\epsilon)}\right)\right. \\
& \geqslant(\beta+o(1)) \log r .
\end{aligned}
$$

Thus, by taking the limit inferior in (2.17), (2.14) follows whenever $0 \leqslant \beta<\infty$. In the case when $\beta=\infty$, choose an arbitrarily large number $\gamma$ instead of $(\beta-\epsilon)$, and then following exactly as above, we get $\nu \geqslant \gamma$. Since $\gamma$ is arbitrary, it follows $\nu=\infty$. This completes the proof of Lemma 4.

LEMMA 5. Let $f(z)=\Sigma_{n=0}^{\infty} a_{n} z^{\lambda_{n}}$ be an entire function of lower $S_{q^{-}}$ order $\nu(0 \leqslant \nu \leqslant \infty)$ such that $\{\psi(n)\}$ forms a nondecreasing function of $n$ for $n>n_{0}$; then $\nu \leqslant \alpha$ where

$$
\alpha \equiv \liminf _{n \rightarrow \infty} \frac{\lambda_{n} \log [q-1]}{\lambda_{n-1}} .
$$

Proof. Since $\{\psi(n)\}$ forms a nondecreasing function of $n$ and $f(z)$ is entire, it follows that $\psi(n)>\psi(n-1)$ for infinitely many values of $n$. Choose $n>n_{0}$ and $\psi(n-1) \leqslant \log r<\psi(n)$; then $m(r)=\left|a_{n}\right| r^{\lambda_{n}}$ and $N(r)=\lambda_{n}$. It follows from Lemma 3, that

$$
N(r)>\exp ^{[q-1]}((\nu-\epsilon) \log r)
$$

for all $r \geqslant r_{0}(\epsilon)$ and $0<\epsilon<\nu<\infty$. Let $\left|a_{m_{1}}\right| z^{\lambda_{m_{1}}}$ and $\left|a_{m_{2}}\right| z^{\lambda_{m_{2}}}$ $\left(m_{1}, m_{2}>n_{0} ; \psi\left(m_{1}-1\right)>r_{0}(\epsilon)\right)$ be any two consecutive maximum terms, then $m_{1} \leqslant m_{2}-1$ and let $m_{1}<n \leqslant m_{2}$. Since $\left|a_{m_{1}}\right| z^{\lambda_{m_{1}}}$ is a maximum term, we have

$$
N(r)=\lambda_{m_{1}} \text { for } \psi\left(m_{1}-1\right) \leqslant \log r<\psi\left(m_{1}\right) .
$$

Then, from (2.19), we have for every $r$ in this interval

In particular,

$$
\lambda_{m_{1}}>\exp ^{[q-1]}((\nu-\epsilon) \log r) \text {. }
$$

$$
N(r)=\lambda_{m_{1}}>\exp ^{[q-1]}\left[\left(\psi\left(m_{1}\right)-C\right)(\nu-\epsilon)\right] \cdot
$$

where $C=\min \left[1,1 / 2\left(\psi\left(m_{1}\right)-\psi\left(m_{1}-1\right)\right)\right]$. Further, we have $\psi\left(m_{1}\right)=$ $\psi\left(m_{1}+1\right)=\cdots=\psi(n-1)$ and so 
$\log \left|a_{n_{0}}\right|+\log \left|a_{n}\right|^{-1}=\sum_{m=n_{0}}^{n}\left(\lambda_{m}-\lambda_{m-1}\right) \psi(m-1)$

$$
\leqslant\left(\lambda_{n}-\lambda_{n_{0}-1}\right) \psi(n-1) \leqslant\left(\lambda_{n}-\lambda_{n_{0}-1}\right)\left(\frac{\log ^{[q-1]} \lambda_{n-1}}{\nu-\epsilon}+C\right) .
$$

This gives, on proceeding to limits, $\nu \leqslant \alpha$, whenever $0<\nu<\infty$. For $\nu=0$ it is obvious and for $\nu=\infty$, it follows exactly on the same lines as above with the same reasonings as in Lemma 4.

By combining Lemmas 3 and 4 we have the following which includes a theorem of Shah [10] and Juneja and Singh [7] for $q=2$.

LEMMA 6. Let $f(z)=\Sigma_{n=0}^{\infty} a_{n} z^{\lambda_{n}}$ be an entire function of lower $S_{q^{-}}$ order $v$ such that $\{\psi(n)\}$ forms a nondecreasing function of $n$ for $n>n_{0}$, then $\nu=\alpha$.

LEMMA 7. Let $f(z)=\sum_{n=0}^{\infty} a_{n} z^{\lambda_{n}}$ be an entire function of lower $S_{q^{-}}$ order $v$, then

$$
\nu \geqslant \gamma=\liminf _{m \rightarrow \infty} \frac{\left(\lambda_{n_{m+1}}-\lambda_{n_{m}}\right) \log [q-1] \lambda_{n_{m}}}{\log \left|a_{n_{m}} / a_{n_{m+1}}\right|}
$$

for any arbitrary sequence of integers $\left\{\lambda_{n_{m}}\right\}_{m=1}^{\infty}$ from $\left\{\lambda_{n}\right\}$ and for the corresponding coefficients $\left\{a_{n_{m}}\right\}$ from $\left\{a_{n}\right\}$ of $f(z)$.

PROOF. For $\gamma=0$ or negative (2.21) is obvious, hence, as usual, first let $0<\gamma<\infty$. Then for all large $m \geqslant m_{0}(\epsilon)$ and $0<\epsilon<\gamma$, we have

$$
\left(\lambda_{n_{m+1}}-\lambda_{n_{m}}\right) \log [q-1] \lambda_{n_{m}}>(\gamma-\epsilon) \log \left|a_{n_{m}}\right| a_{n_{m+1}} \mid \text {. }
$$

Summing the inequalities obtained from (2.22) by replacing $m$ by $n_{0}, n_{0}+$ $1, \cdots, k$, we get

$$
\begin{aligned}
.(\gamma-\epsilon) \log \left|\frac{a_{n_{0}}}{a_{n_{k+1}}}\right| & <\sum_{m=m_{0}}^{k}\left(\lambda_{n_{m+1}}-\lambda_{n_{m}}\right) \log [q-1] \lambda_{n_{m}} \\
& <\left(\lambda_{n_{k+1}}-\lambda_{n_{0}}\right) \log [q-1] \lambda_{n_{k}} .
\end{aligned}
$$

Hence, from Lemma 4 and (2.23), we get $\gamma \leqslant \nu$. The case when $\gamma=\infty$ follows on the same reasonings as those of Lemma 4. This completes the proof of the lemma.

3. In this section we shall prove the following theorems:

THEOREM 1. Let $f(z)=\Sigma_{n=0}^{\infty} a_{n} z^{\lambda_{n}}$ be an entire function of lower $S_{q}$. order $\nu$, then 
and

$$
\nu=\max _{\left\{n_{m}\right\}} \alpha\left(\left\{n_{m}\right\}\right)
$$

$$
\nu=\max _{\left\{n_{m}\right\}} \beta\left(\left\{n_{m}\right\}\right)
$$

where

$$
\alpha\left(\left\{n_{m}\right\}\right)=\liminf _{m \rightarrow \infty} \frac{\lambda_{n_{m}} \log [q-1]}{\lambda_{n_{m-1}}}
$$

and

$$
\beta\left(\left\{n_{m}\right\}\right)=\liminf _{m \rightarrow \infty} \frac{\left(\lambda_{n_{m+1}}-\lambda_{n_{m}}\right) \log [q-1] \lambda_{n_{m}}}{\log \left|a_{n_{m}}\right| a_{n_{m+1}} \mid} .
$$

For $q=2$, the result (3.1) is due to Juneja [6] but the method of proof given here is a different and more elegant one. It is pointed out here that the characterizations (3.1) and (3.2) $\left({ }^{1}\right)$ are complete in the essence of the definition of lower $S_{q}$-order given in the present paper.

Proof. From Lemmas 4 and 7 , it is evident that

$$
\nu \geqslant \max _{\left\{n_{m}\right\}} \alpha\left(\left\{n_{m}\right\}\right)
$$

and

$$
\nu \geqslant \max _{\left\{n_{m}\right\}} \beta\left(\left\{n_{m}\right\}\right) .
$$

Now, consider the function $g(z)=\sum_{s=1}^{\infty} a_{n_{s}} z^{\lambda_{n}}$ where $\left\{\lambda_{n_{s}}\right\}$ denotes the sequence of principal indices of $f(z)$. It is easily seen that $g(z)$ is an entire function and that $f(z)$ and $g(z)$ have the same maximum term and rank for every value of $r$. So, by Lemmas 1 and $3, S_{q}$-order and lower $S_{q}$-order of $g(z)$ are the same as those of $f(z)$. Hence, $g(z)$ is of lower $S_{q}$-order $\nu$. Further, since $\psi\left(n_{s}\right)$ forms a strictly increasing function of $s$, Lemma 6 applied to $g(z)$ gives

$$
\nu=\alpha\left(\left\{n_{s}\right\}\right) .
$$

From (3.5) and (3.7), we get (3.1).

To prove (3.2), we observe that (2.6) gives

$$
\lambda_{n_{s}}>\exp ^{[q-1]}\left[(\nu-\epsilon)\left(\psi\left(n_{s}\right)-C\right)\right]
$$
$q=2$. 
where

$$
C=\min \left[1,1 / 2\left(\psi\left(n_{s}\right)-\psi\left(n_{s-1}\right)\right)\right]
$$

Now, by substituting the value of $\psi\left(n_{s}\right)$ in (3.8), we get

$$
\frac{\left(\lambda_{n_{s+1}}-\lambda_{n_{s}}\right) \log [q-1] \lambda_{n_{s}}}{\log \left|a_{n_{s}} / a_{n_{s+1}}\right|}>(\nu-\epsilon)-\frac{C(\nu-\epsilon)}{\psi\left(n_{s}\right)} .
$$

Taking limit inferior as $s \rightarrow \infty$ in (3.9), we get

$$
\beta\left(\left\{n_{s}\right\}\right) \geqslant \nu \text {. }
$$

Hence, from (3.6) and (3.10) we get equation (3.2) for $f(z)$. This completes the proof of Theorem 1.

The following corollary follows easily from the above theorem.

COROLlary. If $\left\{\lambda_{n_{s}}\right\}$ denotes the sequence of principal indices of $f(z)=$ $\Sigma_{n=0}^{\infty} a_{n} z^{\lambda_{n}}$ and $\rho\left(n_{s}\right)$ denotes the jump points of $N(r)$, then

$$
\nu=\liminf _{s \rightarrow \infty} \frac{\log [q-1]}{\lambda_{n_{s}}} .
$$

For $q=2$, this corollary is due to Gray and Shah [3].

Sato's result (1.6), which gives $S_{q}$-order in terms of the coefficients of the entire series $f(z)=\Sigma_{n=0}^{\infty} a_{n} z^{n}$ may be written as

$$
\lambda=\limsup _{n \rightarrow \infty} \frac{n \log [q-1] n}{\log \left|a_{n}\right|^{-1}}=\max _{\left\{n_{m}\right\}} \alpha^{*}\left(\left\{n_{m}\right\}\right)
$$

where

$$
\alpha^{*}\left(\left\{n_{m}\right\}\right)=\limsup _{m \rightarrow \infty} \frac{n_{m} \log [q-1]}{\log \left|a_{n_{m}}\right|^{-1}}
$$

and $\left\{n_{m}\right\}$ is a subsequence of $\{n\}$ and $\left\{a_{n_{m}}\right\}$ is the corresponding sequence of $\left\{a_{n}\right\}$ from $f(z)$. Thus, our result (3.1) for the lower $S_{q}$-order is an analogue of (1.6) for the case of $S_{q}$-order. Now, we investigate whether a result analogous to (3.2) holds for the case of $S_{q}$-order also, i.e., a relation of the type

where

$$
\lambda=\max _{\left\{n_{m}\right\}} \beta^{*}\left(\left\{n_{m}\right\}\right)
$$

$$
\beta^{*}\left(\left\{n_{m}\right\}\right)=\limsup _{m \rightarrow \infty} \frac{\left(\lambda_{n_{m+1}}-\lambda_{n_{m}}\right) \log [q-1] \lambda_{n_{m+1}}}{\log \left|a_{n_{m}}\right| a_{n_{m+1}} \mid}
$$


holds in the case of $S_{q}$-order. Very simple examples show that this need not hold in general. However in the next theorem, we obtain a result in this direction which holds under some restrictions and at the same time generalizes the results of Shah [10] and Bajpai [1] for $q=2$ and for $q=3,4,5, \cdots$, respectively. These results are valid only when $\lambda_{n}=n$.

THEOREM 2. Let $f(z)=\Sigma_{n=0}^{\infty} a_{n} z^{\lambda_{n}}$ be an entire function of $S_{q}$-order $\lambda_{\text {, }}$ such that $\{\psi(n)\}$ forms a nondecreasing function of $n$ for $n \geqslant n_{0}$, then

$$
\lambda=\limsup _{n \rightarrow \infty} \frac{\left(\lambda_{n+1}-\lambda_{n}\right) \log [q-1] \lambda_{n+1}}{\log \left|a_{n}\right| a_{n+1} \mid} .
$$

Proof. Let

$$
\beta=\limsup _{n \rightarrow \infty} \frac{\left(\lambda_{n+1}-\lambda_{n}\right) \log ^{[q-1]} \lambda_{n+1}}{\log \left|a_{n}\right| a_{n+1} \mid .} .
$$

As usual, first let $\beta>\epsilon>0$, then for all $n \geqslant n_{0}(\epsilon)$, we have

$$
(\beta+\epsilon) \log \left|a_{n} / a_{n+1}\right|>\left(\lambda_{n+1}-\lambda_{n}\right) \log \left[q-1 \dot{j}_{\lambda_{n+1}} .\right.
$$

Summing all the inequalities obtained from (3.12) after writing $\dot{n}=n_{0}, n_{0}+$ $1, \cdots, m$, we get

$$
\begin{aligned}
(\beta+\epsilon) \log \left|a_{n_{0}}\right| a_{m+1} \mid & >\sum_{n=n_{0}}^{m}\left(\lambda_{n+1}-\lambda_{n}\right) \log [q-1] \lambda_{n+1} \\
& \sim \lambda_{m+1} \log ^{[q-1]} \lambda_{m+1} .
\end{aligned}
$$

From (3.13) we easily get

Conversely, when $\{\psi(n)\}$ forms a nondecreasing function of $n$, then by writing

$$
\log \left|a_{n_{0}}\right| a_{n+1} \mid=\left(\lambda_{n_{0}+1}-\lambda_{n_{0}}\right) \psi\left(n_{0}\right)+\cdots+\left(\lambda_{n+1}-\lambda_{n}\right) \psi(n)
$$

we have

$$
\log \left|\frac{a_{n_{0}}}{a_{n+1}}\right| \leqslant\left(\lambda_{n+1}-\lambda_{n_{0}}\right) \psi(n)=\left(\frac{\lambda_{n+1}-\lambda_{n_{0}}}{\lambda_{n+1}-\lambda_{n}}\right) \log \left|\frac{a_{n}}{a_{n+1}}\right| .
$$

Hence, we get

$$
\beta=\limsup _{n \rightarrow \infty} \frac{\left(\lambda_{n+1}-\lambda_{n}\right) \log [q-1]}{\lambda_{n+1}} \mid \lambda .
$$

By combining (3.14) with (3.15) we get (3.11). This completes the proof of the 
theorem. The case $\beta=0$ is obvious and $\beta=\infty$ can be treated similar to $\beta>0$.

As a corollary to Theorem 2, we get the following result of Gray and Shah [3].

COROLLARY. If $\left\{\lambda_{n_{s}}\right\}$ denotes the sequence of principal indices of $f(z)=$ $\Sigma_{n=0}^{\infty} a_{n} z^{\lambda_{n}}$ and $\left\{\rho\left(n_{s}\right)\right\}$ denotes the jump points of $N(r)$, then

$$
\lambda=\limsup _{s \rightarrow \infty} \frac{\log [q-1] \lambda_{n_{s+1}}}{\log \rho\left(n_{s}\right)} .
$$

THEOREM 3. Let $f(z)=\Sigma_{n=0}^{\infty} a_{n} z^{\lambda}$ be an entire function of $S_{q}$-order $\lambda$, then

$$
\nu \leqslant \lambda \liminf _{n \rightarrow \infty} \frac{\log ^{[q-1]} \lambda_{n}}{\log ^{[q-1]} \lambda_{n+1}} .
$$

Further, if $f(z)$ is of $S_{q}$-irregular growth and if $\nu<\mu<\lambda$ then $f(z)$ is of the form $g_{\mu}(z)+h_{\mu}(z)$ where $g_{\mu}(z)$ is of $S_{q}$-order $\leqslant \mu$ and $h_{\mu}(z)=\sum_{p=0^{a}}^{\infty} m_{p} z^{\lambda_{m_{p}}}$ satisfies the inequality

$$
\nu \geqslant \mu \liminf _{p \rightarrow \infty} \frac{\log ^{[q-1]} \lambda_{m_{p}}}{\log ^{[q-1]} \lambda_{m_{p+1}}} .
$$

For $q=2$, this result is due to Whittakar [14].

Proof. Let $r_{t}$ be the value at which $N(r)$ jumps from a value $\leqslant \lambda_{n_{t}}$ to a value $\geqslant \lambda_{n_{t}+1}$. Then

$$
\begin{aligned}
\nu & \leqslant \liminf _{t \rightarrow \infty} \frac{\log ^{[q-1]} N\left(r_{t}-0\right)}{\log r_{t}} \\
& \leqslant \lambda \liminf _{t \rightarrow \infty} \frac{\log ^{[q-1]} N\left(r_{t}-0\right)}{\log ^{[q-1]} N\left(r_{t}+0\right)} \leqslant \lambda \liminf _{n \rightarrow \infty} \frac{\log ^{[q-1]} \lambda_{n}}{\log ^{[q-1]} \lambda_{n+1}} .
\end{aligned}
$$

This establishes (3.16). For establishing (3.17), let us define $g_{\mu}(z)=\Sigma^{\prime} a_{n} z^{\lambda_{n}}$, where $\Sigma^{\prime}$ denotes the summation over $n$, for which

$$
\left(\log [q-2] \lambda_{n}\right)^{-\lambda_{n} / \mu} \geqslant\left|a_{n}\right| \text {. }
$$

Then $g_{\mu}(z)$ is of $S_{q}$-order $\leqslant \mu$. Further, define

$$
h_{\mu}(z)=f(z)-g_{\mu}(z)=\sum_{p=0}^{\infty} a_{m_{p}} z^{\lambda_{m_{p}}}
$$

where $a_{m_{p}}$ satisfies the inequality complementary to (3.18). Hence 


$$
\begin{aligned}
\frac{\log [q]}{\log r} & \geqslant \frac{\log ^{[q-1]}\left[\log \left|a_{m_{p}}\right|+\lambda_{m_{p}} \log r\right]}{\log r} \\
& \geqslant \frac{\log [q-1]\left[-\left(\lambda_{m_{p}} \mid \mu\right) \log [q-1] \lambda_{m_{p}}+\lambda_{m_{p}} \log r\right]}{\log r} .
\end{aligned}
$$

Write $r_{p}=\left(e \log ^{[q-2]} \lambda_{m_{p}}\right)^{1 / \mu}$, then for $r_{p} \leqslant r \leqslant r_{p+1}$, we have

$$
\frac{\log [q]}{\log r} \geqslant \frac{\log ^{[q-1]} \lambda_{m_{p}}[\mu}{\log r} \geqslant \mu \frac{\log ^{[q-1]} \lambda_{m_{p}}}{\log ^{[q-1]} \lambda_{m_{p+1}}}(1+o(1)) .
$$

Taking limit inferior in (3.19), we ultimately have (3.17). This completes the proof of Theorem 3.

4. In this section we shall obtain the coefficient equivalents of (1.6) which in particular will include the results of Basinger [2] $\left(^{2}\right)$ and generalize a result of Shah [11]. We note that our characterization in terms of the coefficients of (1.6) is not complete but is valid for a wider class of entire functions even when $q=$ 2. We shall further obtain some relations involving type, lower type and coefficients of the power series which will generalize the results of Juneja [5] even for $q=2$. Thus, we prove the following: then

$$
\text { THEOREM 4. Let } f(z)=\Sigma_{n=0}^{\infty} a_{n} z^{\lambda_{n}} \text { be an entire function of } S_{q} \text {-order } \lambda_{\text {, }}
$$

$$
t \geqslant \max _{\left\{n_{m}\right\}} \liminf _{m \rightarrow \infty}\left|a_{n_{m}}\right|^{\lambda / \lambda_{n_{m}}} \log [q-2] \frac{\lambda_{n_{m-1}}}{e \lambda} .
$$

Proof. Let $\left\{n_{m}\right\}$ be a strictly increasing sequence of positive integers. Set

$$
B \equiv B\left(\left\{n_{m}\right\}\right)=\liminf _{m \rightarrow \infty}\left|a_{n_{m}}\right|^{\lambda / \lambda_{n_{m}}} \log [q-2] \frac{\lambda_{n_{m}-1}}{e \lambda}
$$

If $B=0$, then $t \geqslant B$ is obvious. Let $B>\epsilon>0$. Then for all sufficiently large $m$, we have,

$$
\left[(B-\epsilon)\left\{\log [q-2] \lambda_{n_{m-1}} \mid e \lambda\right\}^{-1}\right]^{\left(\lambda_{n_{m}} / \lambda\right)}<\left|a_{n_{m}}\right| .
$$

Now Cauchy's inequality and (4.2) give

$$
r^{\lambda_{n_{m}}}\left[(B-\epsilon)\left\{\log [q-2] \lambda_{n_{m-1}} / e \lambda\right\}^{-1}\right]^{\left(\lambda / \lambda_{n_{m}}\right)^{-1}} \leqslant M(r)
$$

(2) This result was also known to all of us even for $q=2$. 
for all $r$ and all sufficiently large $m$. Let us suppose that

$$
\log P=\lambda_{n_{m}} \log r+\frac{\lambda_{n_{m}}}{\lambda} \log (B-\epsilon)-\frac{\lambda_{n_{m}}}{\lambda} \log [q-1] \frac{\lambda_{n_{m-1}}}{e \lambda} .
$$

Then, if

$$
\log D+\log [q-1] \lambda_{n_{m-1}} \mid e \lambda \leqslant \lambda \log r<\log D+\log [q-1] \lambda_{n_{m}} / e \lambda
$$

where $D=e / B$ if $q=2$ and $D=1 / B$ if $q=3,4,5, \cdots$, we get

(4.4) $\log P \geqslant\left(\lambda_{n_{m}} / \lambda\right) \log [D(B-\epsilon)]>e \log [D(B-\epsilon)] \exp ^{[q-2]} r^{\lambda} / D$.

Hence, from (4.3) and (4.4) we obtain

$$
\frac{\log [q-1] M(r)}{r^{\lambda}}> \begin{cases}B \log (e(B-\epsilon) / B) & \text { if } q=2, \\ B & \text { if } q=3,4,5, \cdots,\end{cases}
$$

for all $r \geqslant r_{0}(\epsilon)$. Hence

$$
\liminf _{r \rightarrow \infty} \frac{\log ^{[q-1]} M(r)}{r^{\lambda}}=t \geqslant B \text { for } q=2,3,4, \cdots \text {. }
$$

Since $t \geqslant B\left(\left\{n_{m}\right\}\right)$ for every sequence $\left\{n_{m}\right\}$, it follows that $t \geqslant$ $\max _{\left\{n_{m}\right\}} B\left(\left\{n_{m}\right\}\right)$. Hence the theorem.

As a corollary to Theorem 4, we get the following result which in particular for $q=2$ is due to Basinger [2].

COROLlary. Let $f(z)=\sum_{n=0}^{\infty} a_{n} z^{\lambda_{n}}$ be an entire function of $S_{q}$-order $\lambda$, such that

$$
\liminf _{n \rightarrow \infty} \frac{\log ^{[q-2]} \lambda_{n-1}}{\log ^{[q-2]} \lambda_{n}}=L \quad(>0),
$$

then

$$
t \geqslant L \liminf _{n \rightarrow \infty}\left|a_{n}\right|^{\lambda / \lambda_{n}} \log [q-2] \lambda_{n} / e \lambda
$$

THEOREM 5. Let $f(z)=\Sigma_{n=0}^{\infty} a_{n} z^{\lambda_{n}}$ be an entire function of $S_{q}$-order $\lambda$, such that $\{\psi(n)\}$ forms a nondecreasing function of $n$ for all $n \geqslant n_{0}$, then

$$
t \leqslant \liminf _{n \rightarrow \infty}\left|a_{n}\right|^{\lambda / \lambda_{n}} \log ^{[q-2]} \lambda_{n} / e \lambda .
$$

For $q=2$, this result is due to Basinger [2].

Proof. First, let $0<t<\infty$. Then, from Lemma 3, for all $r \geqslant r_{0}(\epsilon)$ and $t>\epsilon>0$, we have

$$
\log m(r)>\exp ^{[q-2]}\left\{(t-\epsilon) r^{\lambda}\right\} .
$$


If $a_{n_{1}} z^{\lambda_{n_{1}}}$ and $a_{n_{2}} z^{\lambda_{n_{2}}}$ denote two consecutive maximum terms of $f(z)$, then as $\{\psi(n)\}$ forms a nondecreasing function of $n$, we get, for $n_{1} \leqslant$ $n \leqslant n_{2}-1$, that

$$
\psi\left(n_{2}-1\right)=\psi\left(n_{2}-2\right)=\cdots=\psi(n)=\cdots=\psi\left(n_{1}\right)
$$

and

$$
\left|a_{n}\right| r^{\lambda} \lambda_{n}=\left|a_{n_{2}}\right| r^{\lambda_{n_{2}}} \text { for } r=e^{\psi(n)} \text {. }
$$

Thus, from (4.7)-(4.9) we get

(4.10) $\lambda_{n} \psi(n)+\log \left|a_{n}\right|=\lambda_{n_{2}} \psi\left(n_{2}\right)+\log \left|a_{n_{2}}\right|>\exp ^{[q-2]}\left\{(t-\epsilon) e^{\lambda \psi(n)}\right\}$.

If $B^{*}=\left|a_{n}\right|^{\lambda / \lambda_{n}} \log [q-2] \lambda_{n} / e \lambda$ then from (4.10) we get

$$
B^{*}>e^{-\lambda \psi(n)} \exp \left[\frac{\lambda}{\lambda_{n}} \exp ^{[q-2]}\left\{(t-\epsilon) e^{\lambda \psi(n)}\right\}\right] \log [q-2] \frac{\lambda_{n}}{e \lambda} \text {. }
$$

We note that the minimum value of the function

$$
P(r) \equiv \log [q-2] \frac{\lambda_{n}}{e \lambda} r^{-\lambda} \exp \left[\frac{\lambda}{\lambda_{n}} \exp ^{[q-2]}\left\{(t-\epsilon) r^{\lambda}\right\}\right]
$$

is obtained by solving the following equation

$$
\lambda_{n}=\lambda^{\lambda}(t-\epsilon) \prod_{m=1}^{q-2} \exp ^{[m]}\left\{(t-\epsilon) r^{\lambda}\right\}
$$

where the product $\Pi$ is understood to have a value one when $q=2$. Let $r_{n}$ be the value of $r$, for which this minimum is attained. It is easily seen that

$$
P\left(r_{n}\right) \geqslant\left\{\begin{array}{l}
(t-\epsilon)(1-o(1)) \exp (o(1)) \text { if } q=3,4,5, \cdots, \\
(t-\epsilon) \text { if } q=2 .
\end{array}\right.
$$

From (4.11)-(4.13) for $0<t<\infty$, we get $\lim \inf _{n \rightarrow \infty} B^{*} \geqslant t$. In the case when $t=0,(4.6)$ is obvious, while if $t=\infty$, then (4.11)-(4.13) are obtained by replacing $t$ by an arbitrary large number $t^{*}$, which ultimately by making $t^{*} \rightarrow$ $\infty$, leads to $\lim \inf _{n \rightarrow \infty} B^{*}=\infty$. This completes the proof of the theorem.

By combining the corollary to Theorem 4 with Theorem 5, we get the following theorem, which in particular generalizes a result of Shah [11]. such that

THEOREM 6. Let $f(z)=\sum_{n=0}^{\infty} a_{n} z^{\lambda_{n}}$ be an entire function of $S_{q}$-order $\lambda$,

(i) $\{\psi(n)\}$ forms a nondecreasing function of $n$, and

(ii) $\log ^{[q-2]} \lambda_{n} \sim \log [q-2] \lambda_{n+1}$ as $n \rightarrow \infty$; then $t=\lim _{\inf _{n \rightarrow \infty}}\left|a_{n}\right|^{\lambda / \lambda_{n}} \log [a-2] \lambda_{n} / e \lambda$.

Now we shall prove our main theorem of this section which is a coefficient equivalent of (1.6) for all those entire functions for which the principal indices 
$\left\{\lambda_{n_{s}}\right\}$ satisfy an asymptotic relation $\log ^{[q-2]} \lambda_{n_{s}} \sim \log ^{[q-2]} \lambda_{n_{s+1}}$. It will be of interest to see if this result holds without this restriction.

THEOREM 7. Let $f(z)=\Sigma_{n=0}^{\infty} a_{n} z^{\lambda_{n}}$ be an entire function of $S_{q}$-order $\lambda$ and lower $S_{q}$-type $t$, for which $\left\{\lambda_{n_{k}}\right\}$ denotes the sequence of principal indices. If $\log ^{[q-2]} \lambda_{n_{k}} \sim \log [q-2] \lambda_{n_{k+1}}$, as $k \rightarrow \infty$, then

$$
\cdot t=\max _{\left\{n_{m}\right\}}\left\{\liminf _{m \rightarrow \infty}\left|a_{n_{m}}\right|^{\lambda / \lambda_{n_{m}}} \log [q-2] \frac{\lambda_{n_{m-1}}}{e \lambda}\right\} \text {. }
$$

Proof. Consider the function $g(z)=\sum_{k=0}^{\infty} a_{n_{k}} z^{\lambda_{n_{k}}}$ where $\left\{\lambda_{n_{k}}\right\}$ denotes the sequence of principal indices of $f(z)$. It is easily seen that $g(z)$ is an entire function and that $f(z)$ and $g(z)$ have the same maximum term and rank for every value of $r$. So, by Lemmas 1 to 3 , it follows that $f(z)$ and $g(z)$ have the same $S_{q}$-order $\lambda$ and lower $S_{q}$-type $t$. Since $\left\{\lambda_{n_{k}}\right\}$ denotes the sequence of principal indices it also follows that $\left\{\psi\left(n_{k}\right)\right\}$ is a nondecreasing function of $k$. Thus, from Theorem 6 , we get

$$
t=\liminf _{k \rightarrow \infty}\left|a_{n_{k}}\right|^{\lambda / \lambda_{n_{k}}} \log [q-2] \frac{\lambda_{n_{k-1}}}{e \lambda} .
$$

But from Theorem 4, we have

$$
t \geqslant \max _{\left\{n_{m}\right\}}\left\{\liminf _{m \rightarrow \infty}\left|a_{n_{m}}\right|^{\lambda / \lambda_{n_{m}}} \log [q-2] \frac{\lambda_{n_{m}-1}}{e \lambda}\right\} .
$$

Hence, from (4.14) and (4.15), we get (4.13') for $f(z)$. This completes the proof of the theorem.

THEOREM 8. Let $f(z)=\Sigma_{n=0}^{\infty} a_{n} z^{\lambda}$ be an entire function of $S_{q}$-order $\lambda$, $(0<\lambda<\infty)$, $S_{q}$-type $T$ and lower $S_{q}$-type $t$; then

$$
\eta R \leqslant t \leqslant T \leqslant Q
$$

where

and

$$
\begin{aligned}
& Q=\limsup _{n \rightarrow \infty}\left|\frac{a_{n}}{a_{n-1}}\right|^{\lambda /\left(\lambda_{n}-\lambda_{n-1}\right)} \log [q-2] \frac{\lambda_{n}}{\lambda} \\
& R=\liminf _{n \rightarrow \infty}\left|\frac{a_{n}}{a_{n-1}}\right|^{\lambda /\left(\lambda_{n}-\lambda_{n-1}\right)} \log [q-2] \frac{\lambda_{n-1}}{\lambda}
\end{aligned}
$$

$$
\eta=\left\{\begin{array}{l}
\exp \left[\lim \inf _{n \rightarrow \infty}\left(\lambda_{n-1} / \lambda_{n}\right)-1\right] \text { if } q=2 \\
1 \text { if } q=3,4,5, \cdots
\end{array}\right.
$$


290

S. K. BAJPAI, G. P. KAPOOR AND O. P. JUNEJA

For $q=2$ and $\lambda_{n}=n$ this result is due to Juneja [5].

Proof. First, let $0<R \leqslant Q<\infty$, then for every $\epsilon>0$ and for sufficiently large $n \geqslant n_{0}(\epsilon)$ such that $R>\epsilon>0$, we have

$$
\begin{aligned}
& R-\epsilon<\left|a_{n} / a_{n-1}\right|^{\lambda /\left(\lambda_{n}-\lambda_{n-1}\right)} \log ^{[q-2]} \lambda_{n-1} / \lambda, \\
& Q+\epsilon>\left|a_{n} / a_{n-1}\right|^{\lambda /\left(\lambda_{n}-\lambda_{n-1}\right)} \log ^{[q-2]} \lambda_{n} / \lambda .
\end{aligned}
$$

Writing (4.17) for $m=n_{0}, n_{0}+1, \cdots, n$ and multiplying together, we get

$$
\left(\lambda_{n}-\lambda_{n_{0}-1}\right) \log (R-\epsilon)-\sum_{m=n_{0}}^{n}\left(\lambda_{m}-\lambda_{m-1}\right) \log [q-1] \frac{\lambda_{m-1}}{\lambda}
$$

$$
\begin{aligned}
& <\lambda \log \left|\frac{a_{n}}{a_{n_{0}-1}}\right| \\
& <\log (Q+\epsilon)-\sum_{m=n_{0}}^{n}\left(\lambda_{m}-\lambda_{m-1}\right) \log [q-1] \frac{\lambda_{m}}{\lambda} .
\end{aligned}
$$

Now, if $q=3,4,5, \cdots$, and $n(t)=\lambda_{m}$, if $\lambda_{m-1}<t \leqslant \lambda_{m}$, then

$$
\begin{aligned}
& -\sum_{m=n_{0}}^{n}\left(\lambda_{m}-\lambda_{m-1}\right) \log [q-1] \frac{\lambda_{m-1}}{\lambda} \\
& =\lambda_{n_{0}-1} \log [q-1] \frac{\lambda_{n_{0}-1}}{\lambda}-\lambda_{n} \log ^{[q-1]} \frac{\lambda_{n-1}}{\lambda} \\
& +\sum_{m=n_{0}+1}^{n}\left(\log [q-1] \frac{\lambda_{m-1}}{\lambda}-\log [q-1] \frac{\lambda_{m-2}}{\lambda}\right) \lambda_{m-1} \\
& =-\lambda_{n} \log [q-1] \frac{\lambda_{n-1}}{\lambda}+\int_{\lambda_{n_{0}+1}}^{\lambda_{n-1}} n(t) d \log [q-1] \frac{t}{\lambda}+\lambda_{n_{0}-1} \log [q-1] \frac{\lambda_{n_{0}-1}}{\lambda} \\
& >-\lambda_{n} \log [q-1] \frac{\lambda_{n-1}}{\lambda}+\frac{\lambda_{n-1}-\lambda_{n_{0}+1}}{\prod_{m-1}^{q-2} \log ^{(m)} \lambda_{n-1 / \lambda}}+\lambda_{n_{0}-1} \log (q-1) \frac{\lambda_{n_{0}-1}}{\lambda} \\
& =-\lambda_{n} \log (a-1) \frac{\lambda_{n-1}}{\lambda}+o\left(\lambda_{n}\right) \text {. }
\end{aligned}
$$

And, if $q=2$, then for all $n \geqslant n_{0}(\epsilon)$, we have

$$
\begin{aligned}
-\sum_{m=n_{0}}^{n}\left(\lambda_{m}-\lambda_{m-1}\right) \log \frac{\lambda_{m-1}}{\lambda} \\
>-\lambda_{n} \log \frac{\lambda_{n-1}}{\lambda}+\left(\lambda_{n-1}-\lambda_{n_{0}+1}\right)+o\left(\lambda_{n}\right) \\
>-\lambda_{n} \log \frac{\lambda_{n-1}}{\lambda}+\lambda_{n}(\log \eta+1-\epsilon)+o\left(\lambda_{n}\right) .
\end{aligned}
$$


Further,

$$
\begin{aligned}
-\sum_{m=n_{0}}^{n}\left(\lambda_{m}\right. & \left.-\lambda_{m-1}\right) \log [q-1] \frac{\lambda_{m}}{\lambda} \\
= & -\lambda_{n} \log [q-1] \frac{\lambda_{n}}{\lambda}+\lambda_{n_{0}-1} \log [q-1] \lambda_{n_{0}} \\
& +\sum_{m=n_{0}+1}^{n}\left(\log [q-1] \frac{\lambda_{m}}{\lambda}-\log ^{[q-1]} \frac{\lambda_{m-1}}{\lambda}\right) \lambda_{m-1} \\
= & -\lambda_{n} \log [q-1] \frac{\lambda_{n}}{\lambda}+\lambda_{n_{0}-1} \log [q-1] \lambda_{n_{0}} \\
& +\int_{\lambda_{n_{0}+1}^{\lambda_{n}} n(t) d \log [q-1] \frac{t}{\lambda}}
\end{aligned}
$$

where $n(t)=\lambda_{m-1}$ if $\lambda_{m-1} \leqslant t<\lambda_{m}$. Then, if $q=3,4,5, \cdots$

$$
\begin{aligned}
& I=\int_{\lambda_{n_{0}+1}}^{\lambda_{n}} n(t) d \log [q-1] \frac{t}{\lambda} \\
& =\left(\int_{\lambda_{n_{0}+1}}^{\left[\sqrt{\lambda}_{n}\right]}+\int_{\left[\sqrt{\lambda_{n}}\right]}^{\lambda_{n}}\right) n(t) d \log [q-1] \frac{t}{\lambda}
\end{aligned}
$$

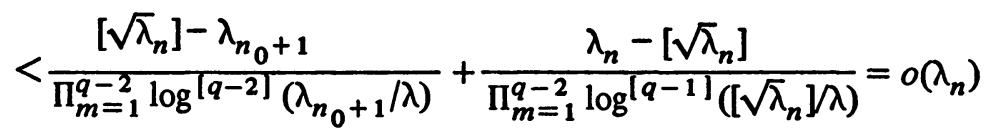

and for $q=2$, we get

$$
I=\int_{\lambda_{n_{0}+1}}^{\lambda_{n}} \frac{n(t)}{t} d t<\left(\lambda_{n}-\lambda_{n_{0}+1}\right) .
$$

From (4.18)-(4.23) we get (4.16) whenever $0<R \leqslant Q<\infty$. In the case when $R=0,(4.16)$ is obvious. If $R=\infty$, then (4.18)-(4.23) are obtained exactly as above by replacing $R-\epsilon$ by any arbitrary large number $R^{*}$, which ultimately leads to $t=\infty$. In the case when $Q=\infty$, then (4.16) is obvious. This completes the proof of Theorem 8 .

THEOREM 9. Let $f(z)=\Sigma_{n=0}^{\infty} a_{n} z^{\lambda_{n}}$ be an entire function of $S_{q}$-order $\lambda$ and $S_{q}$-type $T$ such that $\{\psi(n)\}$ forms a nondecreasing function of $n$; then

$$
\left\{\begin{array}{l}
T \leqslant Q \leqslant e T, \quad \text { if } q=2, \\
T=Q \text { if } q=3,4,5, \cdots .
\end{array}\right.
$$


In the case when $\lambda_{n}=n$ and $q=2$, Theorem 8 is due to Juneja [5].

Proof. If $T=\infty$, then (4.24) is obvious in view of Theorem 3. Let $0 \leqslant T<\infty$. Then, for every $\epsilon>0$, and for sufficiently large $n \geqslant n_{0}(\epsilon)$, we have

$$
\frac{\lambda}{\lambda_{n}} \log \left|a_{n}\right|<\log (T+\epsilon)-\log [q-1] \frac{\lambda_{n}}{e \lambda}
$$

Writing

$$
\begin{aligned}
\log \left|a_{n}\right| & =\log \left|a_{n_{0}}\right|-\sum_{m=n_{0}}^{n-1}\left(\lambda_{m+1}-\lambda_{m}\right) \psi(m) \\
& >\log \left|a_{n_{0}}\right|-\left(\lambda_{n}-\lambda_{n_{0}}\right) \psi(n-1) \quad \text { (by hypothesis) } \\
& =\log \left|a_{n_{0}}\right|+\left(\frac{\lambda_{n}-\lambda_{n_{0}}}{\lambda_{n}-\lambda_{n-1}}\right) \log \left|\frac{a_{n}}{a_{n-1}}\right| .
\end{aligned}
$$

Hence, from (4.25) and (4.26), we get

(4.27) $\log (T+\epsilon)>\log [q-1] \frac{\lambda_{n}}{e \lambda}+\frac{\lambda}{\lambda_{n}}\left[\log \left|a_{n_{0}}\right|+\left(\frac{\lambda_{n}-\lambda_{n_{0}}}{\lambda_{n}-\lambda_{n-1}}\right) \log \left|\frac{a_{n}}{a_{n-1}}\right|\right]$

for all $n \geqslant n_{0}(\epsilon)$. By taking exponentiation of (4.27), we have

$$
\cdot(T+\epsilon)>\left|a_{n_{0}}\right|^{\lambda / \lambda_{n}}\left|\frac{a_{n}}{a_{n-1}}\right|^{\lambda\left(\lambda_{n}-\lambda_{n_{0}}\right) / \lambda_{n}\left(\lambda_{n}-\lambda_{n-1}\right)} \log [q-2] \frac{\lambda_{n}}{e \lambda}
$$

for all $n \geqslant n_{0}(\epsilon)$. Now, by taking limit superior, we ultimately get

$$
T \geqslant \limsup _{n \rightarrow \infty}\left|\frac{a_{n}}{a_{n-1}}\right|^{\lambda /\left(\lambda_{n}-\lambda_{n-1}\right)} \log [q-2] \frac{\lambda_{n}}{e \lambda} .
$$

Thus, by combining (4.16) with (4.28), we get (4.24) whenever $0 \leqslant T<\infty$. This completes the proof of the theorem.

REMARK. It is evident from (4.16) that if $\log ^{[q-2]} \lambda_{n-1} \sim \log ^{[q-2]} \lambda_{n}$ and $0<R=Q<\infty$, then $f(z)$ is of perfectly $S_{q}$-regular growth, of $S_{q}$-order $\lambda$ and $S_{q}$-type $Q$. However the converse of this need not be true in general as can be seen from the following example. If $f(z)=e^{z^{2}}+e^{z}$ then $f(z)$ is of $S_{q}$-order, $\lambda=\nu=2, T=t=1$ and $R=0$ while $Q=\infty$.

5. In this section we shall prove some theorems involving the coefficients of the entire series and growth numbers. It will be seen that these theorems generalize the results of Juneja [4] even for $q=2$. Further, we shall also prove a decomposition theorem for entire functions which are of $S_{q}$-regular growth but 
not of perfectly $S_{q}$-regular growth. Such a theorem is not known even for $q=2$.

THEOREM 10. Let $f(z)=\Sigma_{n=0}^{\infty} a_{n} z^{\lambda_{n}}$ be an entire function of $S_{q}$-order $\lambda, S_{q}$-growth number $\mu$ and lower $S_{q}$-growth number $\delta$ such that $\psi(n)$ forms a strictly increasing function of $n$, then

$$
\mu=B Q \text { and } \delta=B R
$$

where $B=\lambda$ if $q=2, B=1$ if $q=3,4,5, \cdots$, and $Q$ and $R$ are defined as in Theorem 8 .

This theorem generalizes an earlier result of Juneja [4] which is valid only when $\lambda_{n}=n$ and $q=2$.

PrOOF. Since $\{\psi(n)\}$ forms a strictly increasing sequence of $n$, the $\lambda_{n}$ th term will be the maximum term for $|z|=r$, if and only if,

$$
N(r)=\lambda_{n} \text { and } m(r)=\left|a_{n}\right| r^{\lambda_{n}}
$$

for $\psi(n-1) \leqslant \log r<\psi(n)$. Then, for given $\epsilon>0$, and for sufficiently large $n \geqslant n_{0}(\epsilon)$, we have

$$
(\mu-\epsilon)<r^{-\lambda} \log [q-2] \lambda_{n}
$$

and

$$
(\delta+\epsilon)>r^{-\lambda} \log { }^{[q-2]} \lambda_{n}
$$

for all $r$ satisfying $\psi(n-1) \leqslant \log r<\psi(n)$. Then from (5.3) and (5.4)

$$
\begin{aligned}
& (\mu-\epsilon)<\left|a_{n} / a_{n-1}\right|^{\lambda /\left(\lambda_{n}-\lambda_{n-1}\right)} \log [q-2] \lambda_{n}, \\
& (\delta+\epsilon) \geqslant\left|a_{n+1} / a_{n}\right|^{\lambda /\left(\lambda_{n+1}-\lambda_{n}\right)} \log [q-2] \lambda_{n} .
\end{aligned}
$$

Hence on proceeding to limits, we get

$$
\mu \leqslant B R \text { and } \delta \geqslant B Q \text {. }
$$

Further, from (1.7) we have $\log ^{[q-2]} N(r)<(\mu+\epsilon) r^{\lambda}$ for a sequence of values $r=r_{1}, r_{2}, \cdots, r_{n} \rightarrow \infty$. Hence, (5.2), for $n$ 's corresponding to these values of $r_{n}$ 's, yields

$$
\log [q-2] \lambda_{n}<(\mu+\epsilon) r_{n}^{\lambda}<(\mu+\epsilon)\left|a_{n} / a_{n+1}\right|^{\lambda /\left(\lambda_{n+1}-\lambda_{n}\right)} .
$$

This gives

$$
B R \leqslant \mu .
$$

Similarly, it can be shown that 


$$
B Q \geqslant \delta
$$

From (5.6)-(5.8) we get (5.1).

THEOREM 11. Let $f(z)=\Sigma_{n=0}^{\infty} a_{n} z^{\lambda_{n}}$ be an entire function of $S_{q}$-irregular growth, then $t=\mu=R=0$.

For $q=2$, this result is due to Srivastava and Singh [12]. Proof of this theorem is easy so we omit it.

THEOREM 12. Let $f(z)=\Sigma_{n=0}^{\infty} a_{n} z^{\lambda_{n}}$ be an entire function of $S_{q}$-order $\lambda, S_{q}$-growth number $\delta(<\infty)$, lower $S_{q}$-growth number $\mu$ and $S_{q}$-type $T$, and lower $S_{q}$-type $t$; then

$$
\mu \leqslant \delta \liminf _{n \rightarrow \infty} \frac{\log ^{[q-2]} \lambda_{n}}{\log ^{[q-2]} \lambda_{n+1}} .
$$

Further, if $0<t<\eta<T(<\infty)$, then $f(z)$ can be written in the form $f(z)=$

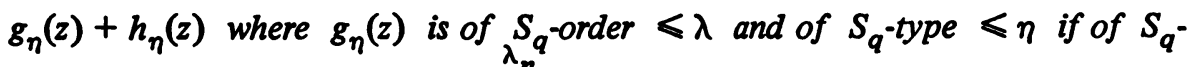
order $\lambda$, and $h_{\eta}(z)=\sum_{p=0}^{\infty} a_{n_{p}} z^{\lambda_{n}}$ satisfies the inequality

$$
t \geqslant \eta \liminf _{p \rightarrow \infty} \frac{\log [q-2] \lambda_{n_{p}}}{\log ^{[q-2]} \lambda_{n_{p+1}} .}
$$

Proof. Let $r_{t}$ be the value at which $N(r)$ jumps from a value $\leqslant \lambda_{n_{t}}$ to a value $\geqslant \lambda_{n_{t}+1}$. Then

$$
\begin{aligned}
\mu & \leqslant \liminf _{t \rightarrow \infty} \frac{\log ^{[q-2]} N\left(r_{t}-0\right)}{r_{t}^{\lambda}} \\
& \leqslant \limsup _{t \rightarrow \infty} \frac{\log ^{[q-2]} N\left(r_{t}+0\right)}{r_{t}^{\lambda}} \liminf _{t \rightarrow \infty} \frac{\log ^{[q-2]} N\left(r_{t}-0\right)}{\log ^{[q-2]} N\left(r_{t}+0\right)} \\
& \leqslant \delta \liminf _{n \rightarrow \infty} \frac{\log ^{[q-2]} \lambda_{n}}{\log ^{[q-2]} \lambda_{n+1}} .
\end{aligned}
$$

This proves the first part of the theorem. For proving the second part, let $g_{\eta}(z)=$ $\Sigma^{\prime} a_{n} z^{\lambda_{n}}$ where $\Sigma^{\prime}$ denote the summation over those $n$ 's for which

$$
\left|a_{n}\right| \leqslant\left(\eta / \log [q-2] \lambda_{n} / e \lambda\right)^{\lambda_{n} / \lambda}
$$

Then, $g_{\eta}(z)$ is of $S_{q}$-order $\leqslant \lambda$, and $S_{q}$-type $\leqslant \eta$ if $g_{\eta}(z)$ is of $S_{q}$-order $\lambda$. Define 


$$
h_{\eta}(z)=f(z)-g_{\eta}(z)=\sum_{p=0}^{\infty} a_{n_{p}} z^{\lambda_{n}}
$$

where $a_{n_{p}}$ 's satisfy the reverse of the inequality (5.11). If $M\left(r, h_{\eta}\right)=$ $\max _{|z|=r}\left|h_{\eta}(z)\right|$, then

$$
\begin{aligned}
& \log ^{[q-1]} M\left(r, h_{\eta}\right)>\log ^{[q-1]}\left\{\left|a_{n_{p}}\right| r^{\lambda n_{p}}\right\} \\
& \geqslant \log [q-2]\left[\frac{\lambda_{n_{p}}}{\lambda} \log \eta-\frac{\lambda_{n_{p}}}{\lambda} \log [q-1] \frac{\lambda_{n_{p}}}{e \lambda}+\lambda_{n_{p}} \log r\right]
\end{aligned}
$$

for all $r$ and $p$. Let $\log r_{p}=X+\lambda^{-1} \log [q-1] \lambda_{n_{p}} / e \lambda$, then, if $r_{p} \leqslant r \leqslant$ $r_{p+1}$, we get

$$
\begin{aligned}
\log ^{[q-1]} M\left(r, h_{\eta}\right) & \geqslant \log ^{[q-2]}\left[\left(X+\lambda^{-1} \log \eta\right) \lambda_{n_{p}}\right] \\
& =\frac{\left(e^{-X} r_{p+1}\right)^{\lambda} \log ^{[q-2]}\left[\left(X+\lambda^{-1} \log \eta\right) \lambda_{n_{p}}\right]}{\log ^{[q-2]}\left(\lambda_{n_{p+1}} / e \lambda\right)} .
\end{aligned}
$$

From this we get

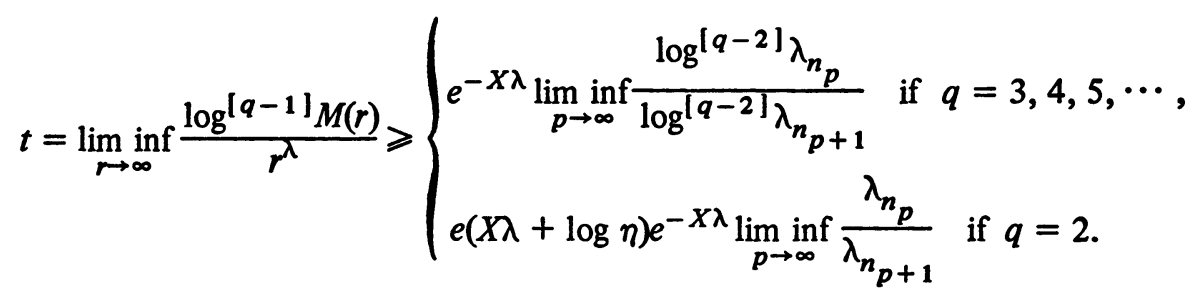

By choosing

$$
X= \begin{cases}-\lambda^{-1} \log \eta & \text { if } q=3,4,5, \cdots \\ (1-\log \eta) / \lambda & \text { if } q=2,\end{cases}
$$

we get (5.10). This completes the proof of the theorem.

6. We have seen in Theorem 11 that if $f(z)$ is of $S_{q}$-irregular growth then lower $S_{q}$-type is zero. In such a case it is natural to seek a constant $\rho(0<$ $\nu \leqslant \rho<\lambda)$ for which

$$
\liminf _{r \rightarrow \infty} \frac{\log ^{[q-1]} M(r)}{r^{\rho}}=A_{\rho}
$$

and $A_{\rho}$ is finite and nonzero. We shall call $A_{\rho}$ to be $S_{q}$ - $\rho$-type for the entire function $f(z)$ and whenever $\rho=\nu$ this constant will be called $S_{q}-\nu$-type. For 
$q=2$, this concept is due to Srivastava and Singh [12]. Coefficient equivalent of $(6.1)$ is as follows:

THEOREM 13. Let $f(z)=\Sigma_{n=0}^{\infty} a_{n} z^{\lambda_{n}}$ be an entire function of $S_{q}$-p-type $A_{\rho}$, then

$$
A_{\rho}=\max _{\left\{n_{m}\right\}}\left\{\liminf _{m \rightarrow \infty}\left|a_{n_{m}}\right|^{\rho / \lambda_{n_{m}}} \log [q-2] \frac{\lambda_{n_{m}-1}}{e \rho}\right\}
$$

provided the principal indices $\left\{\lambda_{n_{s}}\right\}$ satisfy the asymptotic relation $\log ^{[q-2]} \lambda_{n_{s}} \sim$ $\log ^{[q-2]} \lambda_{n_{s+1}}$ as $s \rightarrow \infty$.

We omit the proof of this theorem as it follows on the same lines as the proof of Theorem 7.

Analogues of Theorems 4 to 6 can also be obtained in terms of $A_{\rho}$.

Acknowledgments. Authors express their sincere thanks to the referee for his valuable suggestions which led to our paper in the present form. Authors are also thankful to Professor R. S. L. Srivastava for his interest and encouragement in the present work.

\section{REFERENCES}

1. S. K. Bajpai, On entire functions of fast growth, Rev. Roumaine Math. Pures Appl. 16 (1971), 1159-1162. MR 46 \#3787.

2. R. C. Basinger, On the coefficients of an entire series with gaps, J. Math. Anal.

Appl. 38 (1972), 790-792; See also: J. Reine Angew. Math. 203 (1971), 74. MR 46 \#2056.

3. A. Gray and S. M. Shah, Holomorphic functions with gap power series, Math. Z. 86 (1965), 375-394. MR 33 \#7501.

4. O. P. Juneja, Some properties of entire functions, Dissertation, Indian Institute of Technology, Kanpur, 1965.

5. - On the coefficients of an entire series of finite order, Arch. Math. (Basel) 21 (1970), 374-378. MR 43 \#519.

6. - On the coefficients of an entire series, J. Analyse Math. 24 (1971), 395401. MR 43 \#7632.

7. O. P. Juneja and P. Singh, On the growth of an entire series with gaps, J. Math. Anal. Appl. 30 (1970), 330-334. MR 40 \#7451.

8. O. P. Juneja and G. P. Kapoor, On the lower order of entire functions, J. London Math. Soc. 5 (1972), 310-312.

9. D. Sato, On the rate of growth of entire functions of fast growth, Bull. Amer. Math. Soc. 69 (1963), 411-414. MR 26 \#3903.

10. S. M. Shah, On the lower order of integral functions, Bull. Amer. Math. Soc. 52 (1946), 1046-1052. MR 8, 322.

11. - On the coefficients of an entire series of finite order, J. London Math. Soc. 26 (1952), 45-46. MR 12, 399.

12. R. S. L. Srivastava and P. Singh, On the $\lambda$-type of an entire function of irregular growth, Arch. Math. 17 (1966), 342-346. MR 34 \#1505.

13. G. Valiron, Lectures on general theory of integral functions, Chelsea, New York, 1949. 
14. J. M. Whittakar, The lower order of integral functions, J. London Math. Soc. 8 (1933), 20-27.

DEPARTMENT OF MATHEMATICS, INDIAN INSTITUTE OF TECHNOLOGY, DELHI-29, NEW DELHI, INDIA

DEPARTMENT OF MATHEMATICS, INDIAN INSTITUTE OF TECHNOLOGY, KANPUR-16, INDIA

Current address (G. P. Kapoor): V. V. Post Graduate College, Shamli, Muzaffarnagar, India 